\title{
Outcomes of eight patients after surgical valve replacement for carcinoid heart disease.
}

\author{
Dharmesh RAMLUGUN${ }^{1}$, Michel KINDO ${ }^{1}$, and Jean-Philippe MAZZUCOTELLI ${ }^{1}$ \\ ${ }^{1}$ Strasbourg University Hospital
}

March 5, 2021

\begin{abstract}
Background and aim

Carcinoid heart disease is a rare, acquired heart valvular disease affecting mainly the right chambers. Cardiac surgery is the only definite symptom relieving option and has significantly transformed patient short and midterm prognosis. In this study, we aim to retrospectively analyze the postoperative outcomes of patients with carcinoid syndrome after heart valve surgery.
\end{abstract}

\section{Methods}

Data was retrospectively collected for patients referred to our institution for carcinoid heart disease and operated between 2013 and beginning of 2021 .

\section{Results}

8 patients underwent heart valve replacement with 1 having quadruple valve surgery, 3 patients had both tricuspid and pulmonary valves changed while 4 had only tricuspid valve replacements ; 3 patients had mechanical prostheses. 3 deaths occurred during the follow-up till date with 2 inside the perioperative period. No case of valvular complications was reported. Mean ICU and hospital stay were 9,4 days (range 1-39) and 23,5 days (range 10-52) respectively. 2 patients needed postoperative dialysis, 1 needed re-intubation and no patient required extracorporeal life support. The mean time period from diagnosis to valve surgery was 16,6 months (range 1-60). Follow-up (2-84 months) showed excellent postoperative clinical status with NYHA 1 in all patients.

\section{Conclusions}

Timely heart valve surgery in patients with close multidisciplinary monitoring is associated with good midterm survival rates and low perioperative valvular complications. Patients should be considered for surgery the soonest possible irrespective of the number of valves concerned as functional status improves drastically in most patients.

\section{Hosted file}

Article Carcinoid.pdf available at https://authorea.com/users/398375/articles/511012outcomes-of-eight-patients-after-surgical-valve-replacement-for-carcinoid-heart-disease 